\title{
Testing the Recovery of Intrinsic Galaxy Sizes and Masses of $z \sim 2$ Massive Galaxies Using Cosmological Simulations
}

\author{
Sedona H. Price ${ }^{1}$, Mariska Kriek ${ }^{1}$, Robert Feldmann ${ }^{1,2}$, Eliot Quataert $^{1}$, Philip F. Hopkins ${ }^{3}$, \\ Claude-André Faucher-Giguère ${ }^{4}$, Dušan Kereš ${ }^{5}$, and Guillermo Barro ${ }^{1}$ \\ ${ }^{1}$ Department of Astronomy, University of California, Berkeley, CA 94720, USA; sedona@berkeley.edu \\ ${ }^{2}$ Institute for Computational Science, University of Zurich, CH-8057 Zurich, Switzerland \\ ${ }^{3}$ TAPIR 350-17, California Institute of Technology, Pasadena, CA 91125, USA \\ ${ }^{4}$ Department of Physics and Astronomy and CIERA, Northwestern University, Evanston, IL 60208, USA \\ ${ }^{5}$ Center for Astrophysics and Space Sciences, University of California, San Diego, CA 92093, USA \\ Received 2017 May 9; revised 2017 June 23; accepted 2017 July 3; published 2017 July 18
}

\begin{abstract}
Accurate measurements of galaxy masses and sizes are key to tracing galaxy evolution over time. Cosmological zoom-in simulations provide an ideal test bed for assessing the recovery of galaxy properties from observations. Here, we utilize galaxies with $M_{*} \sim 10^{10}-10^{11.5} M_{\odot}$ at $z \sim 1.7-2$ from the MassiveFIRE cosmological simulation suite, part of the Feedback in Realistic Environments (FIRE) project. Using mock multi-band images, we compare intrinsic galaxy masses and sizes to observational estimates. We find that observations accurately recover stellar masses, with a slight average underestimate of $\sim 0.06$ dex and a $\sim 0.15$ dex scatter. Recovered half-light radii agree well with intrinsic half-mass radii when averaged over all viewing angles, with a systematic offset of $\sim 0.1$ dex (with the half-light radii being larger) and a scatter of $\sim 0.2$ dex. When using color gradients to account for mass-to-light variations, recovered half-mass radii also exceed the intrinsic half-mass radii by $\sim 0.1$ dex. However, if not properly accounted for, aperture effects can bias size estimates by $\sim 0.1$ dex. No differences are found between the mass and size offsets for star-forming and quiescent galaxies. Variations in viewing angle are responsible for $\sim 25 \%$ of the scatter in the recovered masses and sizes. Our results thus suggest that the intrinsic scatter in the mass-size relation may have previously been overestimated by $\sim 25 \%$. Moreover, orientation-driven scatter causes the number density of very massive galaxies to be overestimated by $\sim 0.5$ dex at $M_{*} \sim 10^{11.5} M_{\odot}$.
\end{abstract}

Key words: galaxies: evolution - galaxies: high-redshift - galaxies: structure

\section{Introduction}

Tracing the evolution of galaxy stellar masses and sizes across multiple cosmological epochs provides direct constraints on the growth of galaxies. Recent photometric studies have probed stellar masses for large galaxy samples out to $z \sim 3$ (e.g., Tomczak et al. 2014) and for small samples out to $z \sim 9$-11 (e.g., Oesch et al. 2013). Deep, high-resolution Hubble Space Telescope (HST) imaging has also provided measurements of rest-frame optical sizes for large samples of galaxies out to $z \sim 2.5$ (e.g., van der Wel et al. 2014; Peth et al. 2016). Together, these measurements make it possible to trace the evolution of the mass-size relation (Shen et al. 2003) out to $z \sim 2.5$ (van der Wel et al. 2014).

Despite their central role in galaxy evolution studies, it is uncertain how well measured masses and sizes reflect the intrinsic properties of galaxies. Recovered galaxy properties may be impacted by complex dust-to-star geometry and projection effects. Furthermore, galaxy sizes are often measured from the stellar light distribution, even though light does not directly trace stellar mass in most galaxies. Half-light radii are larger than half-mass radii for many galaxies (Wuyts et al. 2012). Color gradients can be used to estimate half-mass radii (e.g., Szomoru et al. 2013), but it is unclear how accurately they reflect the intrinsic galaxy sizes.

Evaluating parameter recovery requires a galaxy sample with known intrinsic properties. Mock observations of simulated galaxies are ideally suited to this task, as cosmological simulations now probe the complex star, gas, and dust geometry in the interstellar medium with high (sub-kiloparsec scale) resolution (e.g., Hopkins et al. 2014; Schaye et al. 2014; Vogelsberger et al. 2014; Feldmann et al. 2016). Recent studies have investigated the recovery of stellar masses (e.g., Wuyts et al. 2009; Hayward \& Smith 2015; Torrey et al. 2015) and sizes (e.g., Wuyts et al. 2010; Snyder et al. 2015a, 2015b; Taghizadeh-Popp et al. 2015; Bottrell et al. 2017) using mock observations. However, these studies have not simultaneously included dust, multiple viewing angles, high spatial resolution, observational point-spread functions (PSFs), and noise to test parameter recovery in high-redshift galaxies.

In this Letter, we present a study of the recovery of galaxy masses and sizes using mock observations over multiple projections of $z \sim 2$ galaxies from MassiveFIRE (Feldmann et al. 2016), following the same procedures used for observations. Throughout this work, we adopt a $\Lambda$ CDM cosmology with $\Omega_{m}=0.3, \Omega_{\Lambda}=0.7$, and $H_{0}=70 \mathrm{~km} \mathrm{~s}^{-1} \mathrm{Mpc}^{-1}$.

\section{Mock Observations}

We use simulations from the FIRE project (Hopkins et al. 2014) to constrain how well intrinsic galaxy properties can be recovered from observations. Specifically, we analyze the MassiveFIRE suite of cosmological zoom-in simulations (Feldmann et al. 2016, 2017), focusing on a sample of 50 massive galaxy snapshots. We consider all massive $\left(M_{*} \sim 10^{10}-10^{11.5} M_{\odot}\right)$ central and satellite galaxies from the high-resolution runs, using snapshots at both $z \sim 1.7$ and $z \sim 2$ of 21 galaxies (Series A and B in Feldmann et al. 2017) and at $z \sim 2$ of 8 galaxies (Series C in Feldmann et al. 2017, including 4 unpublished galaxies). The sample includes large 

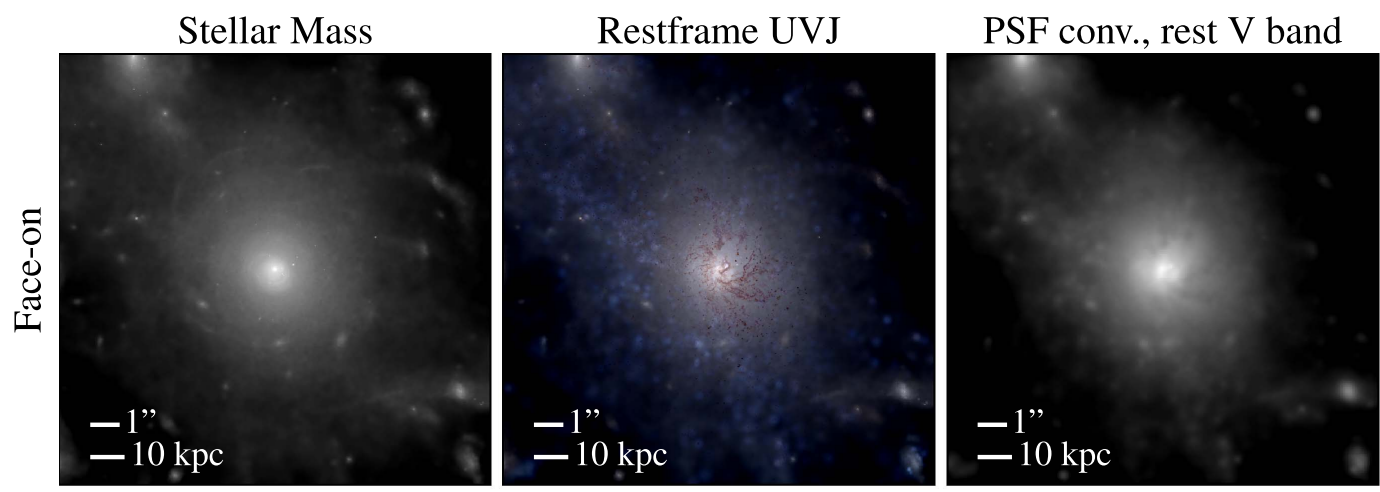

Mock obs, rest V band
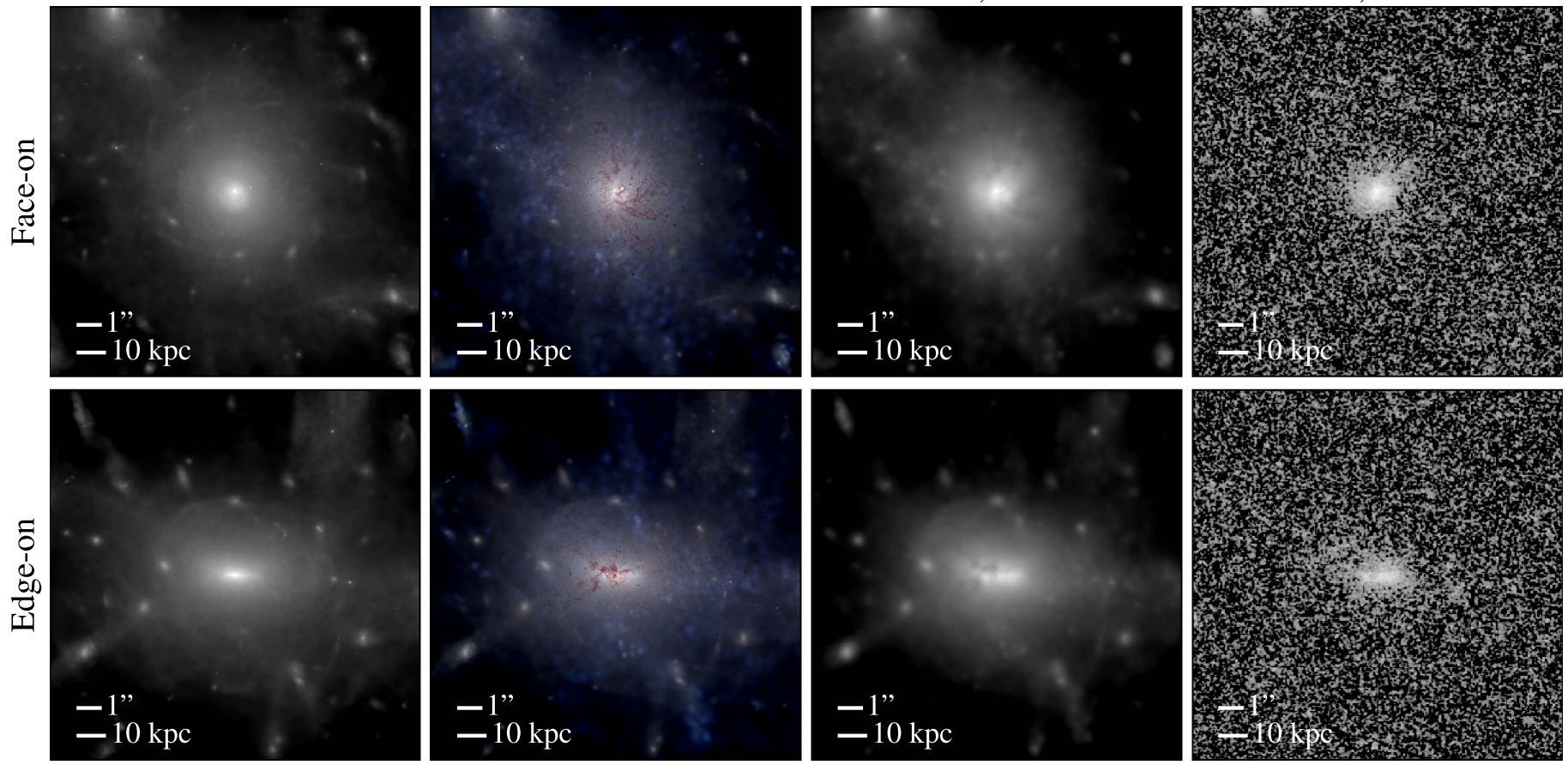

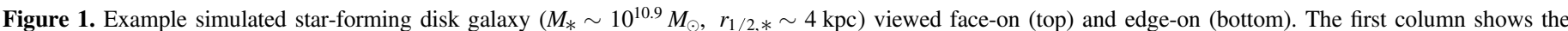

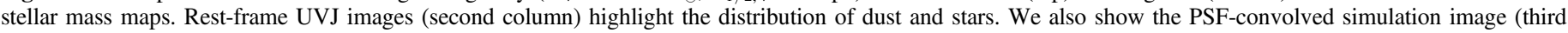
column) and the resulting mock observation (fourth column) for the rest-frame V band. Each image is $144 \mathrm{kpc}$ on each side.

star-forming disks, irregular star-forming galaxies, and quiescent galaxies.

We construct mock multi-filter images of the galaxies using the method described below. To understand how viewing angle affects measurements, we generate images of each galaxy along 25 different projections. First, we generate noise-free multifilter rest-frame images for each projection of each simulated galaxy. Every stellar particle is assigned the spectral energy distribution (SED) of a simple stellar population based on its mass, age, and metallicity using the Bruzual \& Charlot (2003) stellar population synthesis (SPS) models assuming a Chabrier (2003) initial mass function (IMF). Dust attenuation is incorporated by tracing the amount of dust along the line of sight, assuming a Calzetti et al. (2000) curve. Dust content is inferred from the gas particle masses and metallicities, assuming a fixed dust-to-metal ratio. Scattering is indirectly applied by using an empirical dust attenuation curve. Dust emission is omitted as we do not sample the SEDs at long wavelengths. We then sample the dust-attenuated SED in a set of rest-frame filters to obtain mock rest-frame images.

The images are artificially redshifted to the snapshot redshift $(z=2.02$ and 1.67$)$ by applying cosmological dimming, adjusting the angular size, and resampling to match the typical HST/WFC3 drizzled pixel scale (0!"06). The images are convolved with a typical WFC3 PSF (measured from a stack of stars from CANDELS HST/F160W imaging; Skelton et al. 2014). ${ }^{6}$ For simplicity, we apply the same PSF to all bands. Mismatches between the PSFs of different photometric bands can introduce uncertainties in the relative flux calibration. Investigating this uncertainty is beyond the scope of this Letter. However, we note that other studies have investigated the

\footnotetext{
6 Observationally, images are first convolved with the PSF and subsequently sampled within pixels. We find no difference in the mock images when inverting the calculation order.
}

accuracy of flux recovery from low-resolution photometry (e.g., Labbé et al. 2006; Laidler et al. 2007; Wuyts et al. 2008). Finally, we add noise in each band using random CANDELS HST/F160W postage stamps, which contain no detected objects in the 3D-HST catalogs (Skelton et al. 2014) and have typical noise levels. Mock images of each galaxy are constructed for 16 rest-frame filters: ST-UV14, ST-UV17, ST-UV22, ST-UV27 (from Bruzual \& Charlot 2003), SDSS ugriz, U, B, V, R, J, H, and K. Figure 1 shows an example faceon and edge-on view of one galaxy, demonstrating the underlying mass distribution, the rest-frame UVJ colors, the PSF-convolved rest-frame V-band image, and the final mock image including noise.

We detect objects and extract photometry from the mock images following the procedure by Skelton et al. (2014). For every projection of each simulation, we use Source Extractor (Bertin \& Arnouts 1996) in dual-image mode, adopting the parameters used by Skelton et al. and using the rest-frame V-band for detection (roughly covered by F160W at $z \sim 2$ ). The multi-band aperture and total photometry and errors of the objects are determined following Skelton et al. In some projections, dust lanes or bright star-forming clumps lead to multiple detected objects for a single galaxy. To account for this issue, we classify all objects with segmentation maps falling within $2.5 \mathrm{kpc}$ of the galaxy center as part of the galaxy.

\section{Recovering Sizes and Masses}

We measure masses and sizes from the mock images following established observational techniques. Stellar masses $M_{*}$ are determined by fitting the Bruzual \& Charlot (2003) SPS models to all bands of the mock photometry of every object using FAST (Kriek et al. 2009). We assume a Chabrier (2003) IMF, a Calzetti et al. (2000) dust attenuation curve, a delayed 


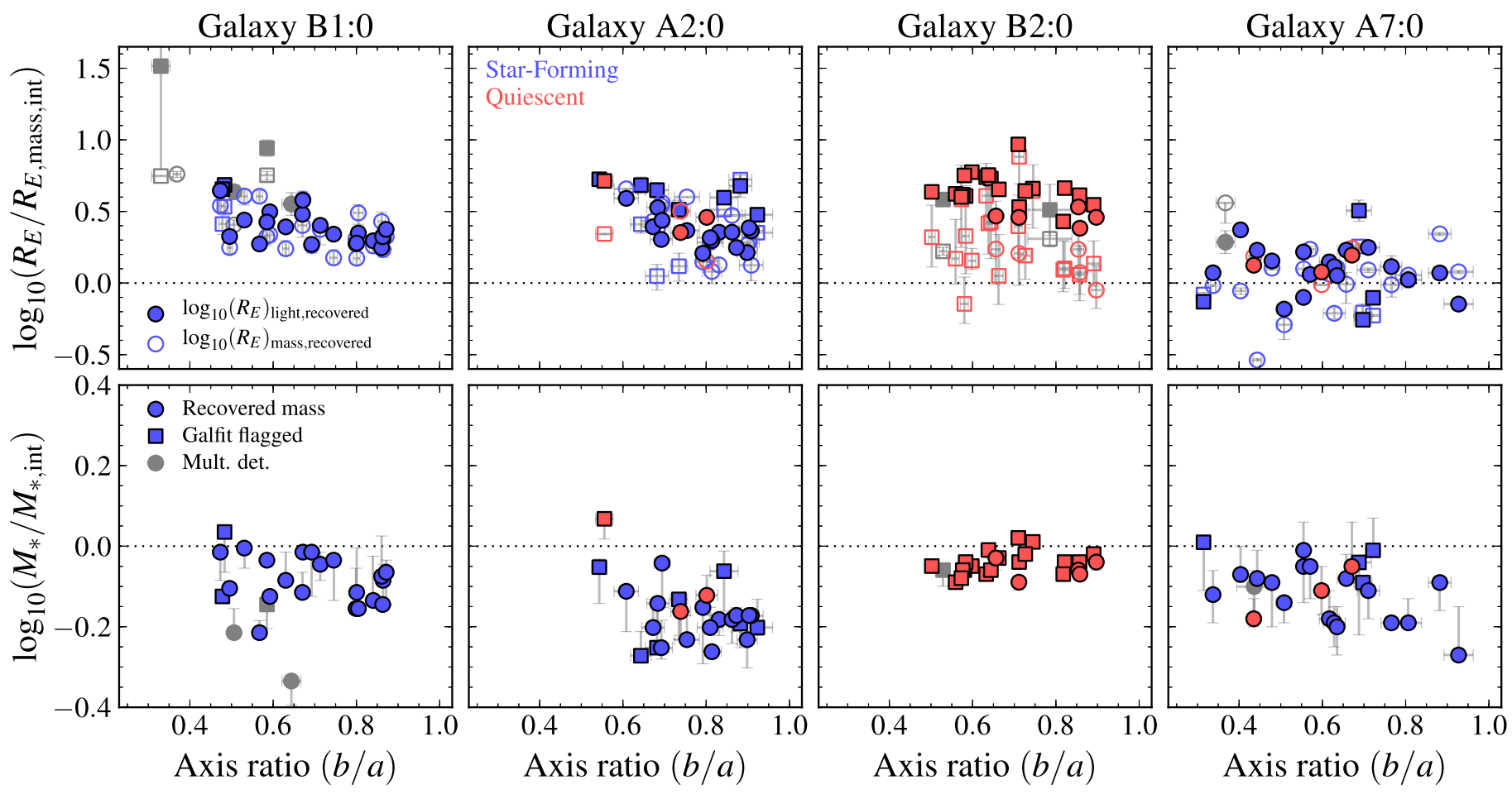

Figure 2. Comparison of recovered and intrinsic sizes and masses over 25 random viewing angles for four simulated MassiveFIRE galaxy snapshots with $M_{*} \sim 10^{10}-10^{11} M_{\odot}$ at $z \sim 2$ as a function of GALFIT axis ratio. In the top row, we show the ratio of the GALFIT half-light radii from the rest-frame V band (filled symbols) and half-mass radii following Szomoru et al. (2013; open symbols) to the intrinsic half-mass radii. In the bottom row, we show the ratio of the recovered and intrinsic masses. Star-forming and quiescent projections (see Section 4) are colored blue and red, respectively. GALFIT-flagged detections are marked with squares. Projections with multiple detections are colored gray, and only the largest radius or mass component is shown. Most orientations for Galaxy B2:0 are flagged due to the Sérsic index reaching the upper limit $(n=8)$.

exponentially declining star formation history, and solar metallicity.

Structural parameters of the simulated galaxies, including the effective radius $R_{E}$, Sérsic index $n$ (Sérsic 1968) and axis ratio $b / a$, are measured using GALFIT (Peng et al. 2010) on the restframe V-band images. We use the GALFIT parameter limits of van der Wel et al. (2012) and flag and exclude from analysis projections for which (a) the GALFIT and V-band total magnitudes differ by $>0.5 \mathrm{mag}$ and (b) fit parameter(s) reached the enforced limit(s). We adopt the semimajor axis $R_{E}$ as the half-light radius. We also estimate half-mass radii following Szomoru et al. (2013). This method uses rest-frame u- and g-band GALFIT profiles and residuals together with an empirical mass-to-light ratio versus color relation to derive a stellar mass profile out to $100 \mathrm{kpc}$.

To determine the fiducial intrinsic masses and sizes of the simulated galaxies, we measure the stellar masses and halfmass radii directly from the mass maps of each galaxy. We define the intrinsic stellar mass for each projection of each galaxy as the mass ${ }^{7}$ enclosed within the Source Extractor Kron ellipse (Kron 1980), masking neighboring detections. Thus, the recovered and intrinsic masses are defined for the same aperture (Skelton et al. 2014). The 2D intrinsic major-axis halfmass radii are defined from growth curves on the projected mass maps, using self-similar ellipses out to the elliptical Kron

\footnotetext{
Bruzual \& Charlot (2003) model masses are used to avoid discrepancies between the recovered and intrinsic masses due to variations in mass-loss prescriptions between the SPS models and the FIRE feedback model (Hopkins et al. 2014), as testing mass-loss variations is beyond the scope of this Letter. These masses are calculated as the current Bruzual \& Charlot (2003) model stellar mass given every star particle's age, initial mass, and metallicity.
}

aperture for each projection as well. We take the median over all projections to obtain the fiducial intrinsic stellar mass and half-mass radius for each galaxy. These intrinsic masses are similar to those derived by Feldmann et al. (2017), which are measured within a sphere of radius $0.1 r_{\text {halo }}$, but the adopted definition allows comparable aperture corrections to be measured from the noise-free light images and recovered mass profiles (see Section 4).

The recovered sizes and masses for four simulated galaxies over 25 random projections are shown in Figure 2. The top panel demonstrates that the measured half-light and half-mass radii are generally larger than the intrinsic radii, while the bottom panel shows that the recovered stellar masses are similar to the intrinsic masses. We observe scatter in both the recovered sizes and masses between different viewing angles. There is a slight trend of increasing radii with decreasing axis ratio $b / a$ for some galaxies, which could be caused by inclination-dependent color gradients. Investigating this trend is beyond the scope of this Letter.

\section{Size and Mass Comparisons}

To understand how well observations recover the sizes and masses of galaxies, we examine the median offset between the recovered and intrinsic sizes and masses for the sample of 50 MassiveFIRE galaxy snapshots, each with 25 projections. Furthermore, we examine whether these offsets differ for starforming and quiescent galaxies. We use the empirical UVJ criterion by Muzzin et al. (2013) at $z>1$ to classify each projection of all galaxies as star-forming or quiescent.

In Figure 3, we show the median recovered half-light and halfmass radii versus intrinsic radii, excluding all GALFIT-flagged 


\section{Recovered half-light radius}
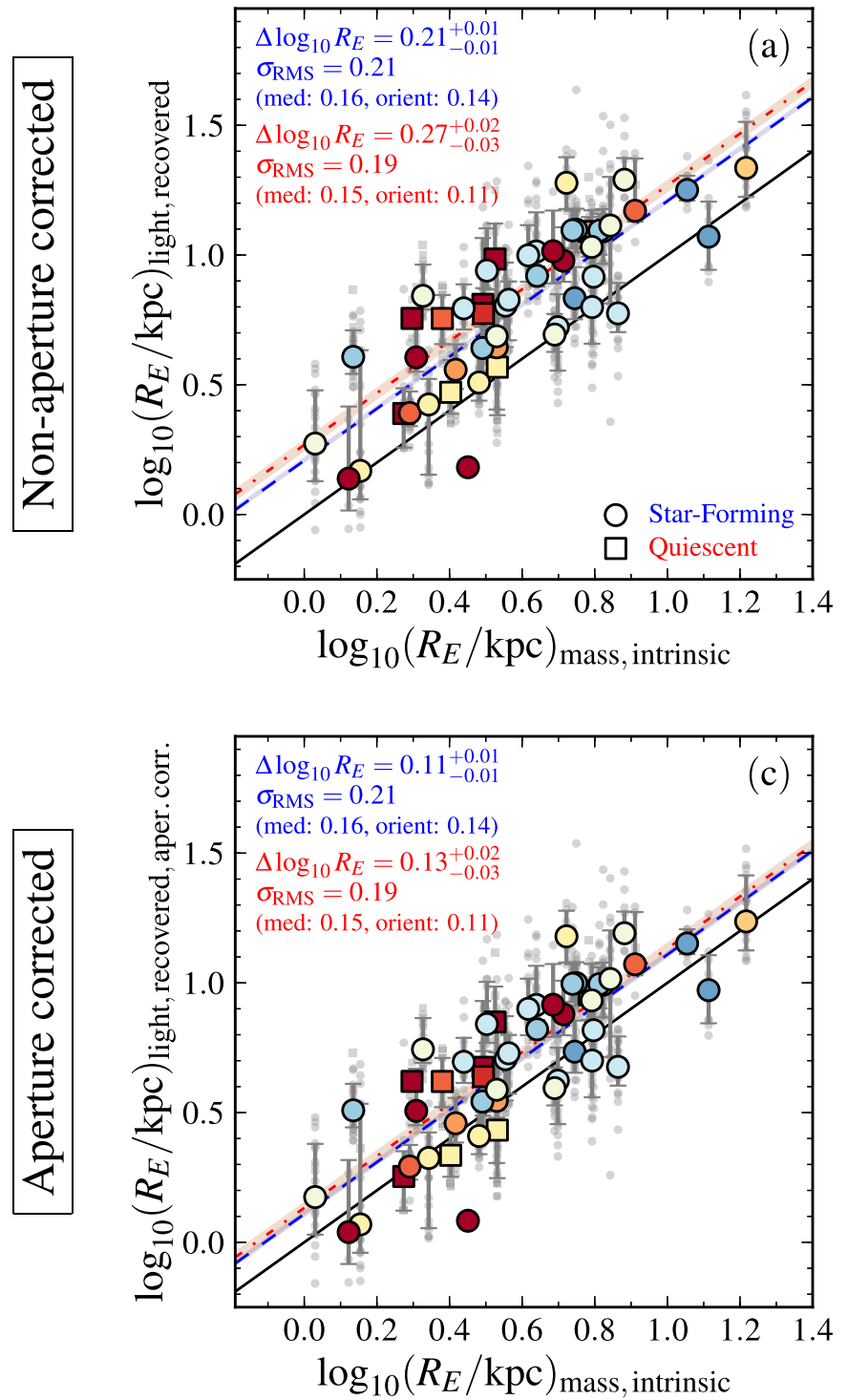

\section{Recovered half-mass radius}
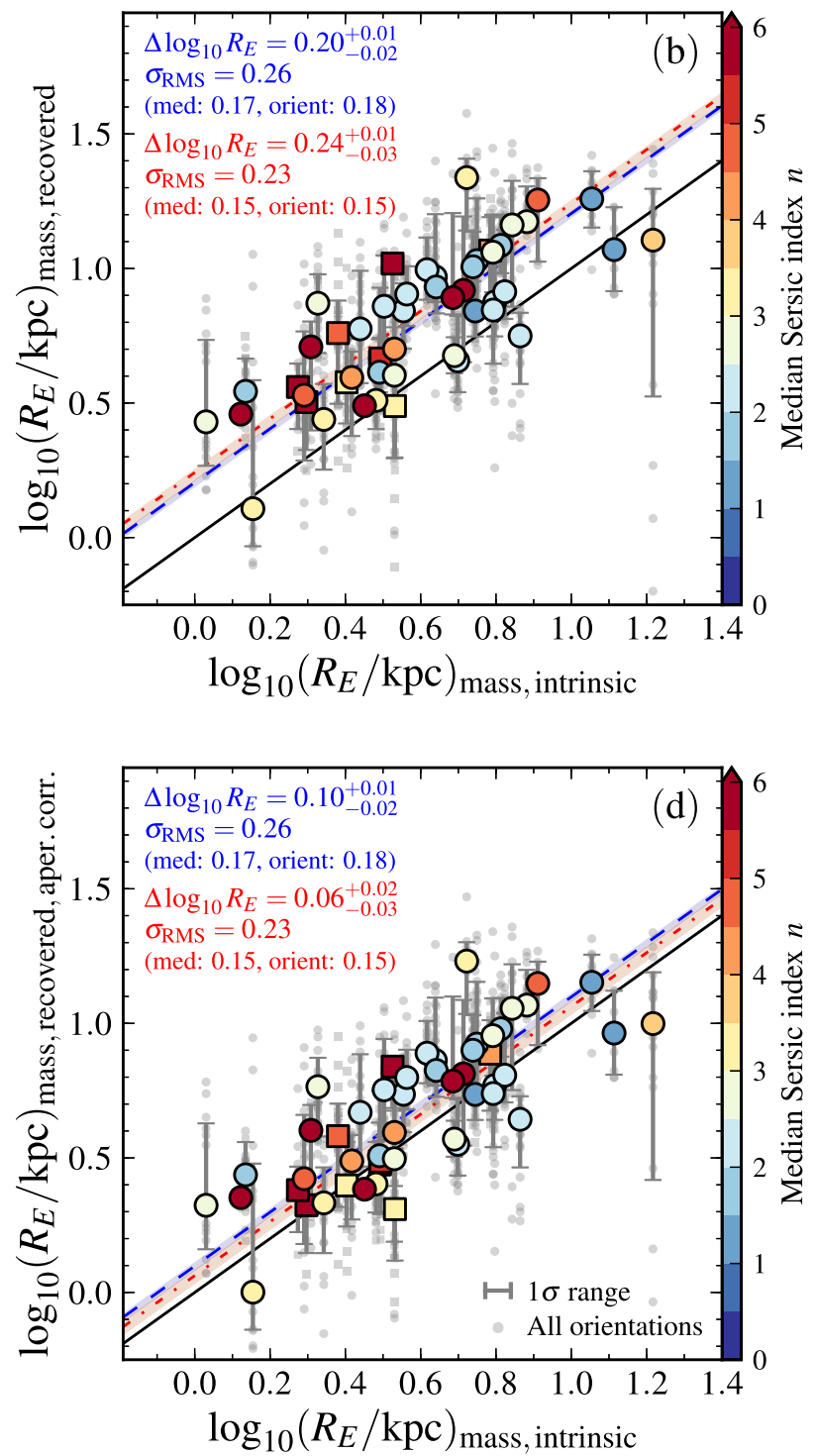

Figure 3. Comparison between intrinsic half-mass radii and the median recovered (a) half-light and (b) half-mass radii, colored by the median (across all orientations) Sérsic index, not accounting for aperture differences. We also compare the intrinsic half-mass radii with aperture-corrected recovered (c) half-light and (d) half-mass radii. Star-forming and quiescent galaxies are shown with circles and squares, respectively. Median U-V and V-J colors are used to categorize each galaxy. GALFITflagged detections are excluded from the median and scatter calculations. The black line shows the one-to-one relationship, and the star-forming and quiescent median size offsets (over all projections) are shown with dashed blue and dashed-dotted red lines, respectively. The shaded regions show the $1 \sigma$ offset uncertainties. The small gray circles (squares) show the radii of all non-flagged star-forming (quiescent) orientations, and the bars denote the $1 \sigma$ range of radii for each galaxy.

detections. We also show all projections and their $1 \sigma$ scatter. We use all unflagged orientations of all galaxies to determine the median offset between the recovered and intrinsic sizes. The offset uncertainties are estimated by bootstrapping the error on the median.

We find that GALFIT radii overestimate the intrinsic radii (Figure 3(a)), with median offsets of $\Delta \log _{10} R_{E}=0.21$ and $0.27 \mathrm{dex}$ for the star-forming and quiescent samples, respectively. The scatter in $\log _{10} R_{E \text {, light,recovered }}$ over all projections for star-forming and quiescent galaxies is $\sigma_{\mathrm{RMS}}=0.21 \mathrm{dex}$ and 0.19 dex, respectively. The scatter of the median sizes of starforming and quiescent galaxies $\left(\left\langle\log _{10} R_{E, \text { light,recovered }}\right\rangle\right.$; weighted by the fraction of unflagged projections) is $\sigma_{\text {med }}=0.16$ and $0.15 \mathrm{dex}$, respectively. Thus, orientation increases the total scatter by $\sigma_{\text {orient }} \sim 0.14$ and $0.11 \mathrm{dex}$ for star-forming and quiescent galaxies, respectively (accounting for measurement errors).

The recovered half-mass radii are also offset from the intrinsic radii (Figure 3(b)), by $\Delta \log _{10} R_{E}=0.20$ and $0.24 \mathrm{dex}$ for the star-forming and quiescent galaxies, respectively. The scatter of $\log _{10} R_{E \text {,mass,recovered }}$ over all projections is slightly larger $\left(\sigma_{\mathrm{RMS}}=0.26\right.$ and $\left.0.23 \mathrm{dex}\right)$, with a similar fraction caused by orientation $(0.18$ and $0.15 \mathrm{dex})$. In comparison to Szomoru et al. (2013), our sample has relatively flat u-g profiles, resulting in similar half-light and half-mass radii.

However, these size comparisons do not account for aperture effects. The intrinsic half-mass radii are defined within finite elliptical apertures (Section 3), whereas GALFIT Sérsic profiles are parametric and integrated out to infinity. To quantify the aperture effects on the measured light-mass size offsets, we 


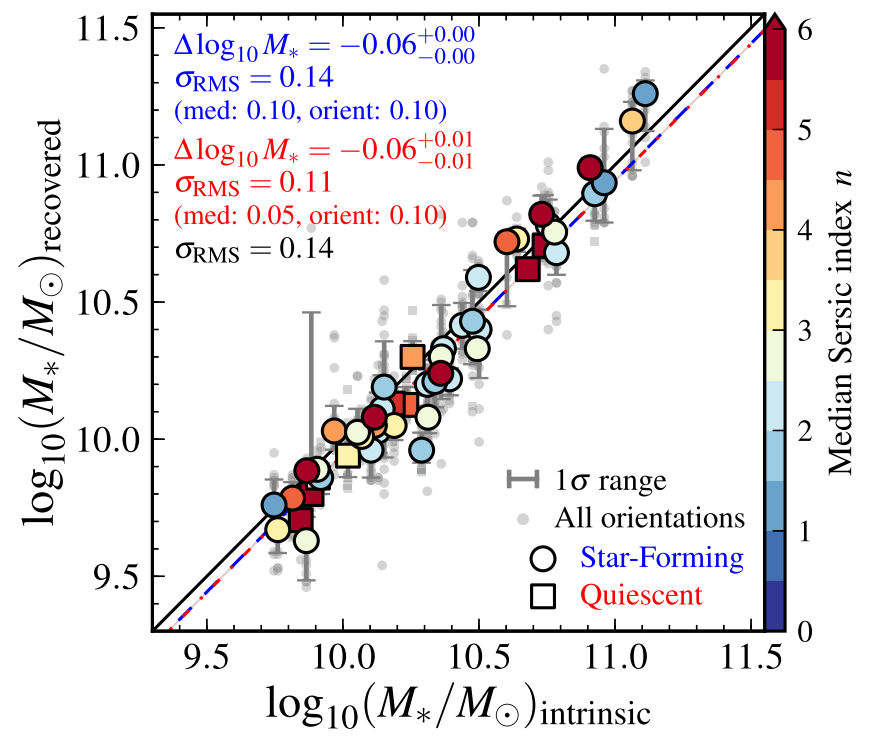

Figure 4. Comparison between intrinsic and median recovered stellar masses, colored by the median Sérsic index. The symbol definitions are the same as in Figure 3, and again GALFIT-flagged detections are excluded. The median offset between the recovered and intrinsic masses is small for both star-forming and quiescent galaxies.

compare GALFIT effective radii to median aperture half-light radii and the recovered half-mass radii to median recovered aperture half-mass radii. Aperture half-light radii are measured directly from the noise-free V-band images, analogous to the half-mass radii measurements. Similarly, recovered aperture half-mass radii are derived from the measured mass profiles. In all cases, aperture effects account for $\sim 0.1 \mathrm{dex}$ of the size offsets.

We find that aperture-corrected half-light radii are in fairly good agreement with the intrinsic half-mass radii (Figure 3(c)), with larger half-light radii by 0.11 dex and 0.13 dex for starforming and quiescent galaxies, respectively, in agreement with previous studies (Wuyts et al. 2010, 2012; Szomoru et al. 2013). The aperture-corrected half-mass and intrinsic half-mass radii have similar systematic offsets $(0.10$ dex and 0.06 dex; Figure 3(d)).

The difference between the aperture-corrected half-light and half-mass radii (Figure 3(c)) appears to be caused by the presence of dust-obscured high central mass concentrations (within $\lesssim 1 \mathrm{kpc}$ ) in many of the galaxies. We would expect that observed color gradients would enable us to recover the central mass component. However, the high central dust content results in saturated color profiles, so this mass component is not recovered using the method of Szomoru et al. (2013; Figure 3(d)). Another potential source of bias between the intrinsic and recovered radii is the use of smooth, single-Sérsic models, as these galaxies have complex structures. Nonetheless, we find that single-Sérsic models introduce little to no bias to the recovered sizes, in agreement with other studies (e.g., Davari et al. 2014, 2016).

Recovered and intrinsic stellar masses are compared in Figure 4, using the same the median calculation method and set of non-flagged detections as for the size comparison. We find that the recovered masses are generally in good agreement with the intrinsic masses, with an offset of only -0.06 dex for both star-forming and quiescent galaxies, and have a scatter of $\sigma_{\mathrm{RMS}}=0.14$ and 0.11 dex over all projections, with 0.10 and 0.05 dex due to orientation effects. Uncertainties in stellar masses can arise from both measured photometry and from mass-to-light ratios derived from SED fitting. We find that photometric uncertainties do not strongly affect the accuracy of the recovered stellar masses. The Source Extractor-derived fluxes recover the intrinsic aperture fluxes very well, with a median fractional flux difference of $-0.3 \%$ and an rms scatter of $7.5 \%$. The small offset and scatter show that stellar masses are recovered well on average over a wide mass range $\left(\sim 10^{9.75}-10^{11.25} M_{\odot}\right)$ and dust attenuation range $\left(A_{\mathrm{V}} \sim 0-2\right)$, but do vary with galaxy viewing direction. Our result of no large systematic mass offset is in good agreement with the findings of other tests of stellar mass recovery using mock observations of simulations (e.g., Wuyts et al. 2009; Torrey et al. 2015).

\section{Discussion and Implications}

Using mock multi-band images of MassiveFIRE simulated galaxies, we show that recovered half-light radii are in good agreement with the intrinsic half-mass radii, with an offset of $\log _{10} R_{E \text {, light,recovered }}-\log _{10} R_{E \text {,mass, intrinsic }} \sim 0.1$ dex (correcting for aperture effects). When we recover half-mass radii by accounting for color gradients due to dust, metallicity, and age, the radii have a similar offset of $\sim 0.1$ dex. Stellar masses are also recovered well on average, with an offset of $\log _{10} M_{* \text {,recovered }}-\log _{10} M_{* \text {,intrinsic }} \sim-0.06$ dex.

By considering the multiple viewing angles of every galaxy, we show that a sizable fraction of the mass and radii scatter is caused by orientation effects. These projection effects may result from the random distribution of bright clumps within a galaxy, a non-uniform or patchy dust distribution, or gradients in metallicity and stellar population age (Kelvin et al. 2012).

We find no systematic differences between the recovery of masses or radii for massive star-forming and quiescent galaxies. Thus, observed differences between star-forming and quiescent galaxy sizes at $z \sim 2$ likely indicate true differences in their stellar mass distributions.

These results have important implications for measuring galaxy structural growth through mass-size relations. First, the mass-size relation zero-point will be systematically overestimated by $\sim 0.1$ dex if half-light radii are used rather than half-mass radii. Second, the intrinsic scatter of the light-based mass-size relation may be overestimated due to random variations in viewing angle, implying the intrinsic mass-size relation could be tighter than previously thought. To quantify the effect of orientation on the mass-size relation scatter, we compare the combined orientation-corrected mass and radius scatters with the combined total scatter. We use the scatter of the medians, $\sigma_{\text {med }}$, as the "intrinsic" scatter (as the mass and radii offsets are uncorrelated), and take the error-corrected RMS scatter as the total scatter, $\sigma_{\text {tot }}=\sqrt{\sigma_{\text {RMS }}^{2}-\sigma_{\text {err }}^{2}}$. The orientation-corrected mass-size relation scatter is $\sim 75 \%$ of the error-corrected total scatter $\left(\sigma_{\text {med }} / \sigma_{\text {tot }}\right)$ for both star-forming and quiescent galaxies.

We illustrate the differences between the observed mass-size relations at $z \sim 1.75$ by van der Wel et al. (2014) and the inferred half-mass radii mass-size relations corrected for orientation effects for both star-forming and quiescent galaxies in Figure 5(a). This figure demonstrates both the zero-point offset due to using intrinsic half-mass versus recovered halflight radii (corrected for aperture effects; Figure 3) and stellar mass recovery (Figure 4), and the reduced intrinsic scatter once orientation effects are corrected. 

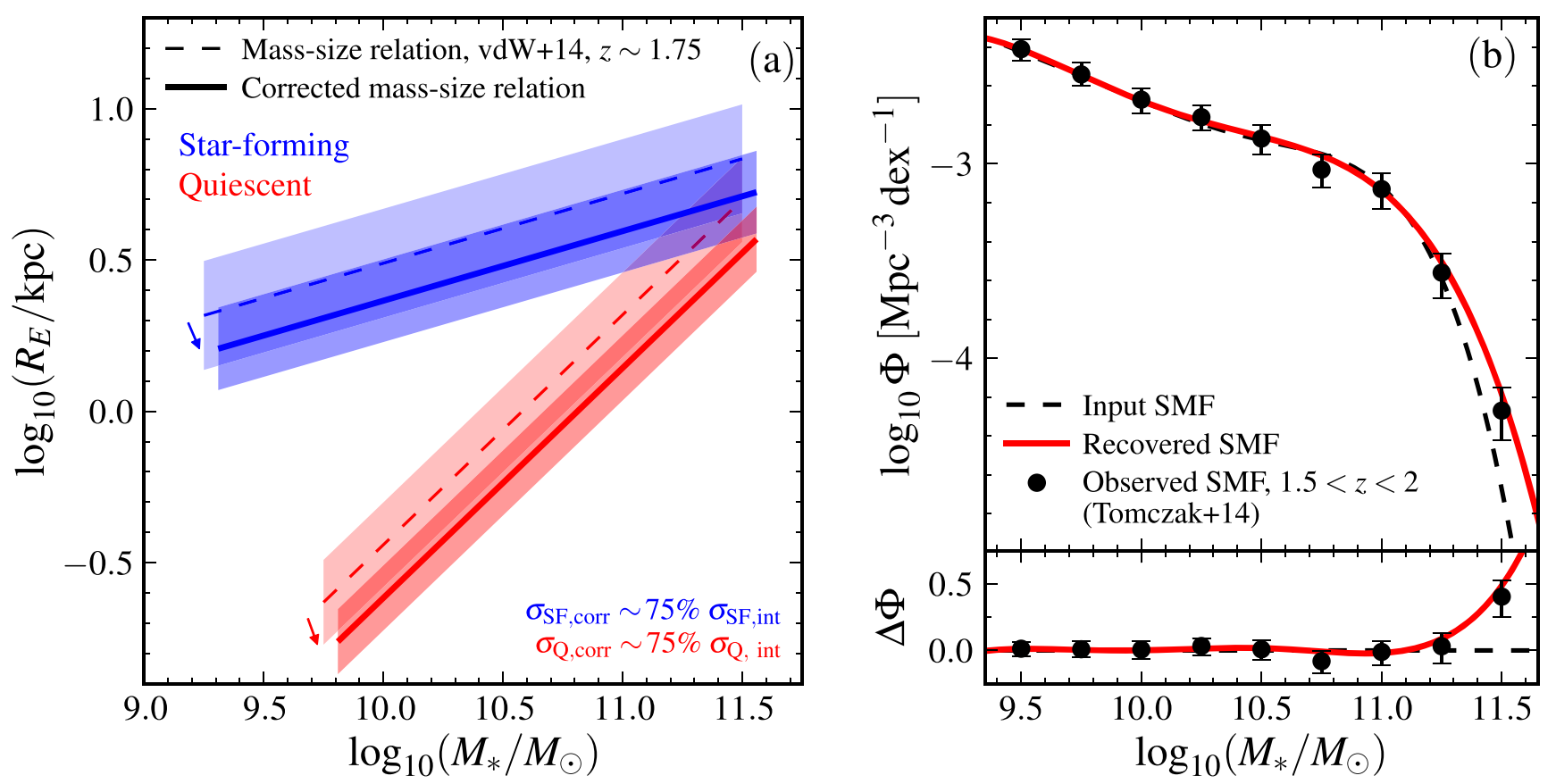

Figure 5. Possible systematic effects on observed galaxy properties at $z \sim 1.7-2$. (a) Shift and scatter reduction of observed mass-size relations. Dashed lines show the star-forming (blue) and quiescent (red) mass-size relations by van der Wel et al. (2014) at $z \sim 1.75$, with light shaded regions showing the observed intrinsic scatter $\sigma \log _{10}\left(R_{\text {eff }}\right)$. Corrected mass-size relations (based on half-mass radii) are shown with solid lines, demonstrating the inferred zero-point offset (Figures 3 and 4). The darker shaded regions represent the intrinsic scatter corrected for orientation effects. (b) Effect of orientation-based scatter on stellar-mass functions (SMFs). The recovered SMF (red line), similar to the SMF at $1.5<z<2$ observed by Tomczak et al. (2014; black circles), deviates from the true SMF (black dashed line) at high masses when mass scatter is applied to a sample drawn from the true SMF.

Even though masses are recovered well on average, the scatter in stellar masses has important implications for studying galaxy populations. For example, scatter impacts the measurement of stellar-mass functions (SMFs). In Figure 5(b), we demonstrate how orientation scatter causes an overestimate of the number density of high-mass galaxies. We draw a galaxy population directly from an input SMF, perturb the masses by the orientation scatter, and then measure the SMF. The input parameters are chosen so the recovered SMF roughly approximates the best-fit $1.5<z<2$ SMF by Tomczak et al. (2014). The true SMF falls off faster than the observed SMF at high masses due to the combination of projectiondriven scatter and the steepness of the SMF at the high-mass end, by up to $\sim 0.5$ dex at $M_{*} \sim 10^{11.5} M_{\odot}$. Hence, many massive galaxies may have such large observed masses as a result of orientation effects. Orientation-driven scatter will also impact other measurements, including the scatter of the starforming main sequence (e.g., Whitaker et al. 2014; Shivaei et al. 2015) and inferred dynamical masses (e.g., Price et al. 2016; Wuyts et al. 2016).

Furthermore, our results demonstrate the difficulty of comparing the sizes of observed and simulated galaxies (see Figure 3(a)). When directly comparing 3D-aperture half-mass radii derived from the simulations and GALFIT effective radii, we find an offset of $\sim 0.2$ dex for both star-forming and quiescent galaxies. To make a fair comparison between observations and simulations, simulated galaxy half-light radii should be measured from mock images using the same methodology applied to observations.

We note the following caveats to this analysis. First, the selected galaxies may not be fully representative of the properties of massive galaxies at $z \sim 1.7-2$. Thus, the measured offsets may not be applicable to all galaxies at these redshifts. Moreover, the relative corrections for star-forming and quiescent galaxies may depend on the realism of the specific simulation models. Finally, we do not account for systematic modeling errors. We have only considered one set of stellar population models and one dust law, applied with a simple line-of-sight attenuation. Modeling choices could affect the recovered offsets and the scatter through systematic color gradient trends and variation in dust attenuation over different viewing angles. Future work is needed to fully understand the impact of dust, non-smooth galaxy morphologies, and specifics of the dust radiative transfer modeling when measuring simulated galaxy properties.

We acknowledge valuable discussions with M. Franx, D. Szomoru, K. Whitaker, C. Hayward, C.-P. Ma, and K. Suess. This work made use of astropy (Robitaille et al. 2013) and pysynphot (Lim et al. 2015). S.P. was supported by a National Science Foundation Graduate Research Fellowship under grant DGE 1106400. M.K. acknowledges support from NSF AAG grant 1313171 and STScI grants AR-13907 and AR-12847, provided by NASA through a grant from the Space Telescope Science Institute. R.F. was supported in part by NASA through Hubble Fellowship grant HF2-51304.001-A awarded by the Space Telescope Science Institute, which is operated by the Association of Universities for Research in Astronomy, Inc., for NASA, under contract NAS 5-26555, by the Theoretical Astrophysics Center at UC Berkeley, and by the Swiss National Science Foundation (grant No. 157591). R.F. and E.Q. acknowledge support from NASA ATP grant 12-ATP-120183. P.H. was supported by an Alfred P. Sloan Research Fellowship, NASA ATP grant NNX14AH35G, and NSF Collaborative Research grant 1411920 and CAREER grant 1455342. C.A.F.G. was supported by NSF grants 
AST-1412836 and AST-1517491, NASA grant NNX15AB22G, and STScI grant HST-AR-14562.001. D.K. acknowledges support from NSF grant AST-1412153 and a Cottrell Scholar Award from the RCSA.

\section{References}

Bertin, E., \& Arnouts, S. 1996, A\&A, 117, 393

Bottrell, C., Torrey, P., Simard, L., \& Ellison, S. L. 2017, MNRAS, 467, 1033

Bruzual, G., \& Charlot, S. 2003, MNRAS, 344, 1000

Calzetti, D., Armus, L., Bohlin, R. C., et al. 2000, ApJ, 533, 682

Chabrier, G. 2003, PASP, 115, 763

Davari, R., Ho, L. C., \& Peng, C. Y. 2016, ApJ, 824, 112

Davari, R., Ho, L. C., Peng, C. Y., \& Huang, S. 2014, ApJ, 787, 69

Feldmann, R., Hopkins, P. F., Quataert, E., Faucher-Giguère, C.-A., \& Kereš, D. 2016, MNRAS, 458, L14

Feldmann, R., Quataert, E., Hopkins, P. F., Faucher-Giguère, C.-A., \& Kereš, D. 2017, MNRAS, 470, 1050

Hayward, C. C., \& Smith, D. J. B. 2015, MNRAS, 446, 1512

Hopkins, P. F., Kereš, D., Oñorbe, J., et al. 2014, MNRAS, 445, 581

Kelvin, L. S., Driver, S. P., Robotham, A. S. G., et al. 2012, MNRAS, 421, 1007

Kriek, M., van Dokkum, P. G., Labbé, I., et al. 2009, ApJ, 700, 221

Kron, R. G. 1980, ApJS, 43, 305

Labbé, I., Bouwens, R., Illingworth, G. D., \& Franx, M. 2006, ApJL, 649, L67

Laidler, V. G., Papovich, C., Grogin, N. A., et al. 2007, PASP, 119, 1325

Lim, P. L., Diaz, R. I., \& Laidler, V. 2015, PySynphot User's Guide (Baltimore, MD: STScI), https://pysynphot.readthedocs.io/en/latest/
Muzzin, A., Marchesini, D., Stefanon, M., et al. 2013, ApJ, 777, 18 Oesch, P. A., Bouwens, R. J., Illingworth, G. D., et al. 2013, ApJ, 773, 75 Peng, C. Y., Ho, L. C., Impey, C. D., \& Rix, H.-W. 2010, AJ, 139, 2097 Peth, M. A., Lotz, J. M., Freeman, P. E., et al. 2016, MNRAS, 458, 963

Price, S. H., Kriek, M., Shapley, A. E., et al. 2016, ApJ, 819, 80 Robitaille, T. P., Tollerud, E. J., Greenfield, P., et al. 2013, A\&A, 558, A33

Schaye, J., Crain, R. A., Bower, R. G., et al. 2014, MNRAS, 446, 521

Sérsic, J. L. 1968, Atlas de Galaxias Australes (Cordoba: Observatorio Astronomico)

Shen, S., Mo, H. J., White, S. D. M., et al. 2003, MNRAS, 343, 978

Shivaei, I., Reddy, N. A., Shapley, A. E., et al. 2015, ApJ, 815, 98

Skelton, R. E., Whitaker, K. E., Momcheva, I. G., et al. 2014, ApJS, 214, 24

Snyder, G. F., Lotz, J., Moody, C., et al. 2015a, MNRAS, 451, 4290

Snyder, G. F., Torrey, P., Lotz, J. M., et al. 2015b, MNRAS, 454, 1886

Szomoru, D., Franx, M., van Dokkum, P. G., et al. 2013, ApJ, 763, 73

Taghizadeh-Popp, M., Fall, S. M., White, R. L., \& Szalay, A. S. 2015, ApJ, 801,14

Tomczak, A. R., Quadri, R. F., Tran, K.-V. H., et al. 2014, ApJ, 783, 85

Torrey, P., Snyder, G. F., Vogelsberger, M., et al. 2015, MNRAS, 447, 2753

van der Wel, A., Bell, E. F., Häussler, B., et al. 2012, ApJS, 203, 24

van der Wel, A., Franx, M., van Dokkum, P. G., et al. 2014, ApJ, 788, 28

Vogelsberger, M., Genel, S., Springel, V., et al. 2014, MNRAS, 444, 1518

Whitaker, K. E., Franx, M., Leja, J., et al. 2014, ApJ, 795, 104

Wuyts, S., Cox, T. J., Hayward, C. C., et al. 2010, ApJ, 722, 1666

Wuyts, S., Förster Schreiber, N. M., Genzel, R., et al. 2012, ApJ, 753, 114

Wuyts, S., Franx, M., Cox, T. J., et al. 2009, ApJ, 696, 348

Wuyts, S., Labbé, I., Schreiber, N. M. F., et al. 2008, ApJ, 682, 985

Wuyts, S., Schreiber, N. M. F., Wisnioski, E., et al. 2016, ApJ, 831, 149 